\title{
Electrocardiographic Alarms in the Acute Care Setting
}

\author{
Jing Jing Zhang, MSN \\ Patricia A. Chin School of Nursing \\ California State University Los Angeles \\ Los Angeles, California USA
}

\author{
Nnenna Weathers, PhD \\ Patricia A. Chin School of Nursing \\ California State University Los Angeles \\ Los Angeles, California USA
}

\begin{abstract}
Electrocardiographic [ECG] alarms are used in acute care settings to continuously monitor patients' cardiac status, and to alert clinicians to potentially life-threatening changes in a patient's condition. However, the numbers of alarms in the acute care setting have increased exponentially in the past 20 years. This was to ensure patient safety, and to avoid adverse events that could end in litigations [1]. Cardiac alarms represent the second most frequent alarm in the acute care setting at $37 \%$ [2]. Although ECG alarms may help save lives, proper alarm management has been problematic and ECG alarms that were designed to enhance patient safety have become a top national safety hazard. Registered Nurses [RNs] must respond to a variety of alarms in acute care settings; however many of these alarms require no action because they may not be triggered by an actual health need. Responding to non-actionable alarms can interrupt nursing workflow, increase the likelihood of clinical errors, and can lead to alarm fatigue and nurse burnout, as well as serious financial consequences for the organizations. This paper discusses problems with ECG alarm management as experienced by RN's, and provides suggestions to improve alarm management in acute care settings.
\end{abstract}

Keywords-alarm management; alarm fatigue, electrocardiographic alarms; acute care setting.

\section{INTRODUCTION}

Electrocardiographic [ECG] alarms are used in the acute care setting to continuously monitor patients' cardiac status, and to alert clinicians to potentially life-threatening changes in a patient's condition. However, the numbers of alarms in the acute care setting have increased exponentially in the past 20 years in order to ensure patient safety, and to avoid adverse events that could end in litigations [1]. Cardiac alarms represent the second most frequent alarm in the acute care setting at 37\%. [2]. Although ECG alarms may help save lives, proper alarm management has been problematic. In a study by the Food and Drugs Administration, 566 deaths occurring from 2005 to 2008 were related to alarm safety [3]. In 2003, the Joint Commission reviewed 23 sentinel incidents, and found through root cause analysis that inappropriate alarm management was the main contributing factor [2]. As a result, the Joint Commission set proper alarm management as a National Patient Safety Goal [2]. Further investigations by the Joint Commission from 2009 to 2012 found 98 sentinel events related to alarm management from 2009 to 2012 [3]. In 2014, the Joint Commission made effective alarm management an accreditation requirement for all hospitals [2].

The ECG alarm that was designed to enhance patient safety has become a top national safety hazard. Instances of problematic ECG alarms include the death of a 60 -year-old man because of a lack of response to the apnea alarm [4]. On another occasion, a cardiac alarm sounded for more than 75 minutes when a non-functional battery apparently contributed to a patient's eventual un-witnessed cardiac arrest [2]. Due to the impact on lives the Joint Commission not only made ECG alarm management a National Patient Safety Goal in 2013, but appropriate alarm control is now an accreditation requirement [5].

Registered Nurses [RNs] must respond to a variety of alarms in acute care settings, however many of these alarms require no action because they may not be triggered by an actual health need. Responding to non-actionable alarms interrupts nursing workflow, increases the likelihood of clinical errors, leads to alarm fatigue, nurse burnout, and insurmountable financial consequences for the organizations. The purpose of this paper is to discuss problems with ECG alarm management as experienced by RN's, and to provide suggestions to improve alarm management in acute care settings.

\section{High ALARM FREQUENCY}

The acute care setting can have as many as 40 alarms on various equipment including respirators, pulse oximetry, arterial pressure and ECG monitors [2]. In one study 350 alarms per day were reported [2] while another study reported a total of 318,000 cardiac alarms for 154 patients over a period of two months [6]. Philips Healthcare, a cardiac alarm manufacturer, conducted an audit on a telemetry unit and found that an RN may have to respond to an average of 3.7 alarms per minute [7]. Regulatory restrictions require default alarm settings to be highly sensitive in order to avoid liability issues in case adverse events occur [7]. Philips Healthcare named failure mode, faulty sensors, loose sensor connection, overly sensitive settings, inaudible alarms due to simultaneous sounds and lack of response from clinicians as main causes of excess telemetry alarms [7]. To avoid missed alarms, alarm sensitivity was increased, thus leading to even more alarms sounding off in an already-noisy environment [7]. Additionally, alarms

DOI: $10.5176 / 2345-7198 \_5.1 .49$

ISSN 2345-7198

CThe Author(s) 2018. This article is published with open access by the GSTF 
sounded do not only indicate changes in patient status, a large portion of them are associated with equipment malfunction including device problems such as a loose lead connection or low battery [2].

\section{ALARM FATIGUE}

The ECG alarm was designed under the "better-safe-thansorry" logic, meaning that alarm, can be highly sensitive but low in specificity [8]. False alarms, or alarms that have no clinical significance occur in up to $99 \%$ of all alarm [8]. These false alarms create a condition where alarms lose credibility causing nurses to respond mostly to alarms they consider critical [2]. Moreover, the design of ECG alarms does not consider the cognitive capacity and processing mechanisms of the healthcare worker [1]. A study at a large Canadian Hospital reported that clinicians were only able to recognize half of the alarms that exist in Intensive Care Units even though they may have worked there on a daily basis for years [9]. Alarms that are often loud, and frequent can result in nurses ignoring them or turning them off. According to a survey by the American College of Clinical Engineering, 81\% of nurses surveyed considered non-actionable alarms disruptive to patient care, $77 \%$ believed false alarms lower alarm credibility, leading to alarm silencing [8].

Overwhelmed nurses can develop alarm fatigue, which is a phenomenon where healthcare workers who are exposed to a high frequency of alarms become desensitized and selectively respond to whatever alarm they deem important [2]. Many nurses suspend or deactivate alarms, which have directly led to untoward patient outcomes [2]. A patient died in a Massachusetts hospital after the ECG alarm was turned off, 3 post-operative patients died in the state of Pennsylvania after the alarm was deactivated, and a 17-year-old patient after a simple tonsillectomy died because her cardiac alarm was muted [10].

\section{NOISE POLlUTION}

Hospital noises can affect both patients and nurses. Hospital noise has been reported as the number one cause of sleep disturbance in hospitals [11]. World Health Organization (WHO) recommends an average noise level of $35 \mathrm{~dB}$ in the hospital; however, it has been reported that patient room noise levels never once fell below the WHO recommended level and daytime peak level reached $80.3 \mathrm{~dB}$ which is an intensity similar to that of a chainsaw [12]. Patients have also reported at least one hour less than their baseline sleep, with low sleep efficiency of $73.3 \%$ while hospitalized. Additionally, $42 \%$ of patients and staff studied reported alarms to be the main noise disruption [12]. The ECG alarms, and excessive noise in general was associated with poor wound healing in patients [13], increased anxiety, stress, and increased pain perception of pain [14]. Hospital noise can also lead to medical communication errors [15], and can cause the development of long-term physiological and psychological problems including burnout [16].
Aside from adverse outcomes for patients and for nurses, hospital noise can have direct financial implications for the organization. The Consumer Assessments of Healthcare Providers and Systems (CAHPS) scores include the care environment as an important indicator of a patient's hospital experience [18]. As hospital noise is part of the care environment CAHPS evaluates noise by asking patients a question on the quietness of their rooms at night [17]. Responses to this question indicate that hospital noise has consistently been the number one patient complaint across the country, and is often the question with the lowest score [18].

\section{EQUIPMENT MANAGEMENT}

Interventions to manage hospital noises include a variety of solutions that have essentially been technology-based [7]. Using high-quality sensors, replacing single-use sensors frequently, and educating staff and patients on ways to minimize signal loss in order to decrease false positives have been suggested [7]. After an alarm management taskforce switched to disposable leads, alarms were reduced by $42 \%$ per bed per day overall [19]. Alarm manufacturers have suggested adjusting bedside ergonomics to prevent cable dislodgement and malfunction [7]. The Joint Commission Requirement also requires that all hospitals regularly maintain and test alarm systems to prevent equipment malfunction [20].

Setting alarms at varying decibels that align with the degree of morbidity may help reduce alarm fatigue [8]. It has been suggested that human illness does not exist in a binary fashion, and cannot be adequately monitored that way causing a need for new smart alarms that can differentiate pathological signs over physiological variables [21]. These researchers suggest that reducing false alarms without compromising sensitivity or specificity may also be helpful; therefore, the use of a multivariable fuzzy temporal profile model that can mitigate cognitive overload thus increasing responses to alarms [21]. Others have suggested using silent alarms to directly notify a specific nurse, or using an alarm grading hierarchy in which only more critical alarms are sounded to help nurses better prioritize alarm responses [22]. Additionally, non-critical alarms can be translated into a message sent directly to a nurse's pager leading to fewer audible alarms, a quieter hospital environment, and reduced alarm fatigue among nurses [7].

Reducing inactionable alarms caused by motion artifact can be helpful in reducing alarm fatigue [8]. Reports indicated that more than $52 \%$ of alarms were the result of patient movement [23]. It has been suggested that introducing a pre-alarm signal delay of 5 to 10 seconds could reduce alarm load by 26\% [24]. Increasing pre-alarm delay to 14 and 17 seconds may also reduce non-actionable alarms by 50 and $57 \%$ respectively [25].

Differentiating ECG alarms from other alarm sounds in acute care settings is another strategy to reduce alarm fatigue [9]. To comply with WHO's recommendation, alarms must be audible and should be tested in their existing environment for audibility [12]. An ideal alarm sound should be easy to learn, 
easy to locate, and hard to be masked by other hospital noises [1]. Proper alarm prioritization needs to be in place so that the intensity of the alert reflects the condition and urgency of the signal [1]. To ensure prompt recognition of the ECG alarm sound and what it indicates the ECG alarm sound should also be standardized to reduce noises that the nurse must learn to recognize [26]. IEC 60601 is a technical standard for medical equipment that has now been adopted as a regulatory framework in the US, and it suggests standardization of alarms for different physiological functions [27]. A relatively new development in alarm technology is the intelligent alarm system in which the alarm has the ability to make simple decisions using the IF-THEN rules or fuzzy logic [21]. Once triggered, the alarm device determines whether the event detected requires an intervention [21].

All ECG monitors have default alarm thresholds suited for the acute care environment, where small changes in patients' status can be closely monitored [7]. The Joint Commission indicated that default ECG parameters could increase the number of false alarms [20]. It has been observed that ECG alarm frequency can be reduced by $50 \%$ when the high heartrate parameter is increased from 120 to 130 beats per minute [28]. Without causing harming, an 89\% reduction in ECG alarms was also observed when heart-rate parameters were slightly widened [29]. Another suggestion is to adjust alarm limits to match meaningful changes in patient status, and using multi-parametric algorithms to identify true critical changes or trends [7]. Individualizing ECG settings could mean for example that heartrate parameter for patients with known atrial fibrillation should be set at a level above 100 in order to reduce the number of non-actionable alarms. In line with this thinking, nurse-led customization of alarm parameters within one hour of assuming care of a patient has been suggested [30]. Others have reported that increasing the premature ventricular contraction limit from 6 to 10 per minute did not interfere with patient safety [8]. The ECG equipment default setting consist of many duplicate alarms [8]. For instance, the alarm sounds twice when the heart rate is low because the high/low heart rate alarm and bradycardia alarm both go off. Changing high/low heart rate alarm to a message level warning, and keeping the bradycardia/tachycardia alarm at warning level showed reduction in duplicate sounds [8].

\section{NURSE AND PATIENT EDUCATION}

Whether RNs are generally proficient at adjusting ECG alarms according to clinical importance is questionable. Without a clear understanding of the features and limitations of ECG equipment, they cannot adequately assess the cause of alarms nor adjust them accordingly. Educating RNs on ECG alarm function may help reduce inactionable alarms [31]. A good understanding of how to review alarm data and trends can also help RNs realize which alarms require immediate attention [31]. Suggestions to improve RNs' knowledge include online education [32]. This is similar to the suggestion by the American Association of Critical Care Nurses that retraining RNs on individualizing alarm parameters may help reduce inactionable alarms [8]. Other sources of false alarms include patient movement and intentional or unintentional removal of sensors. Educating patients in plain and simple terms, and involving family members in the process may improve cooperation with continuous ECG monitoring thus reducing false alarms. Helping patients to understand why they are being monitored, what adverse outcomes require caution and when monitoring can stop may not only decrease alarm frequency but also improve patient satisfaction [33].

\section{OVERMONITORING}

Over-monitoring is another factor that can contribute to excessive alarms [34]. Although the American Heart Association (AHA) has specific standards and indications for ECG monitoring, many patients continue to receive continuous telemetry without an indication [35]. Studies found that eliminating inappropriate ECG monitoring could save hospitals with at least 400 beds up to $\$ 250,000$ per year [35]. While ECG alarms are used to continuously monitor patients' cardiac status, the importance of regular assessments remains invaluable. Studies have reported that hourly rounding increases patient safety and satisfaction [36]. It also decreases inactionable ECG alarms as well as the overall workload, and hence decreases alarm fatigue for RNs [36]. No monitor should replace regular assessments done during hourly rounding [36], and healthcare workers should never fully depend on electronic equipment.

\section{INTERDISCPLINARY COLLABORATION}

Alarm management is a dynamic process that requires constant reevaluation and improvement. The Healthcare Technology Foundation, an interdisciplinary task force formed by The American College of Clinical Engineers believes that improving the safe use of healthcare technology requires collaborative efforts from diverse stakeholders in order to design, use, and service technology, services and equipment [37]. Many current strategies for alarm management are based on studies done by interdisciplinary task forces at various hospitals. The ECRI also underlined the importance of involving RNs in developing alarm management strategies and processes [38].

\section{CONCLUSION}

Alarm management is complex and practices may vary across hospital environments. Although alarm fatigue has been recognized as problematic for both RN's and patients, standardized recommendations on best practices have yet to be put forth [7]. Development of a systematic, evidence-based approach to safe, responsible alarm management is needed [7]. Additionally, ECG education for RN's and patients could be beneficial in reducing alarm fatigue thus enhancing patient care as well as health outcomes. 


\section{REFERENCES}

[1] J. Edworthy, and E. Hellier, "Alarms and human behavior: implications for medical alarms,” Br J Anaesth, vol. 97, pp. 12-17, Jul. 2006.

[2] S. Sendelbach, and M. Funk, "Alarm fatigue: a patient safety concern," AACN Adv Crit Care, vol. 24, pp. 378-86, Oct. 2013.

[3] L. Honan, M. Funk, M. Maynard, D. Fahs, J.T. Clark, and Y. David, "Nurses' perspectives on clinical Alarms," Am J Crit Care, vol. 24, pp. 387-95, Sep. 2015.

[4] T. Purbaugh, "Alarm fatigue: a roadmap for mitigating the cacophony of beeps,” Dimens Crit Care Nurs, vol. 33, pp.4-7, Jan. 2014.

[5] D. M. Korniewicz, T. Clark, and Y. David, “A national online survey on the effectiveness of clinical alarms,” AM J Crit Care, vol. 17, pp.36-41, Jan. 2008.

[6] B. J. Drew, P. Harris, J. K. Zegre-Hernsey, T. Mammone, D. Schindler, R. Salas-Boni, Y. Bai, A. Tinoco, Q. Ding, and X. Hu, "Insights into the problem of alarm fatigue with physiologic monitor devices: a comprehensive observational study of consecutive intensive care unit patients,” PLoS One, vol. 9, pp. 1-23, Oct. 2014.

[7] Philips Healthcare, "Taking alarm management from concept to reality: a step by step guide,” [Online]. Available: https://www.usa.philips.com/b-dam/b2bhc/us/whitepapers/alarmsystems-management/An-action-Plan.pdf. [Accessed: Nov 20, 2016].

[8] K. C. Graham, and M. Cvach, "Monitor alarm fatigue: standardizing use of physiological monitors and decreasing nuisance alarms,” Am J Crit Care, vol. 19, pp. 28-35, Jan. 2010.

[9] K. L. Momtahan, B. W. Tanslet, and R. Hetu, "Audibility and identification of auditory alarms in the operating room and intensive care unit,” Ergonomics, vol. 36, pp. 1159-76, Oct. 1993.

[10] W. Saltzman, "Investigation: dangers of medical recover rooms," Patient Safety Network, Feb 5, 2013. [Online]. Available: http://abclocal.go.com/wpvi/story?section=news/special_reports\&id=89 80647. [Accessed:Oct 10, 2017]

[11] C. A. Cmiel, D. M. Gasser, L. M. Oliphant, and A. J. Neveau, "Noise control: a nursing team's approach to sleep promotion,” Am J Nurs, vol. 104, pp.40-48, Feb. 2004.

[12] J. C. Yoder, P. G. Staisiunas, D. O. Meltzer, K. L. Knutson, and V. M. Arora, "Noise and sleep among adult medical inpatients: Far from a quiet night,” Arch Intern Med, vol. 172, pp. 68-70, Jan. 2012.

[13] T. Hsu, E. Ryherd, K. Persson, and J. Ackerman, "Noise pollution in hospitals: Impact on patients,” JCOM, vol. 19, pp. 301-309, Jul. 2012.

[14] N. Akansel, and S. Kaymakci. "Effects of Intensive Care Unit Noise on Patients: a study on coronary artery bypass graft surgery patients,” J Clin Nurs, vol. 17, pp. 1581-90, Jun. 2008.

[15] A. P. Gurses, and P. Carayon, "Exploring performance obstacles of intensive care nurses,” Appl Ergon, vol. 40, pp. 509-18, May 2009.

[16] A. Mahmood, H. Chaudhury, and M. Valente, "Nurses' perceptions of how physical environment affects medication errors in acute care settings,” Appl Nurs Res, vol. 24, pp. 229-37, Nov. 2011.

[17] S. E. Mazer, "Creating a culture of safety: reducing hospital noise," Biomed Instrum Tehcnol, vol. 46, pp. 350-5, Sep. 2012.

[18] Centers for Medicare and Medicaid Services, "HCAHPS fact sheet," Baltimore, MD, Jun. 2016. [Online]. Available: http://www.hcahpsonline.org/Facts.aspx. [Accessed Jan 20, 2017].

[19] AAMI Foundation HTSI, “Safety Innovations,” 2012. [Online]. Available: http://www.premiersafetyinstitute.org/wpcontent/uploads/Johns-Hopkins-White-Paper.pdf. [Accessed Nov. 11, 2017].

[20] The Joint Commission, “The joint commission announces 2014 National Patient Safety Goal,” Joint Commission Perspectives, vol. 33, no. 7, pp. 1-4. 2013. [Online]. Available: https://www.jointcommission.org/assets/1/18/JCP0713_Announce_New _NSPG.pdf. [Accessed Nov. 17, 2017].
[21] A. Otero, A., P. Felix, P., S. Barro, and F. Palacios, “Addressing the flaws of current critical alarms: a fuzzy constraint satisfaction approach,” Artificial Intelligence in Medicine, vol. 47, pp. 219-38, Nov. 2009.

[22] M.E. Speich, "Reducing alarm fatigue in the intensive care unit: A quality improvement research study,” Doctoral Dissertations, 1449, May 2017.

[23] M.C. Chambrin, D. Calvelo-Aros, A. Jaborska, C. Chopin, and B. Boniface, "Multicentric study of monitoring alarms in the adult intensive care unit (ICU): a descriptive analysis,” Intesive Care Medicine,” Intensive Care Med, vol. 25, pp.1360-1366, Dec. 1999.

[24] E.M. Koski, T. Sukuvaara, A. Makivirta, and A. Kari, “A knowledgebased alarm system for monitoring cardiac operated patients assessment of clinical performance,” Int J Clin Monit Comput, vol. 11, pp. 79-83, May. 1994.

[25] M. Borowski, M. Gorges, R. Fried, O. Such, C. Wrede, and M. Imhoff, "Medical device alarms," Biomed Tech (Berl), vol 56, pp. 73-84, Apr. 2011.

[26] M. Cvach, “Monitor alarm fatigue: An integrative review,” Biomedical Instrum Technol, vol. 46, pp. 268-77, Jul. 2012.

[27] International Organization for Standardization, “IEC 60601-1-8:2006,” International Electrotechnical Comission, vol. 2, pp. 1-144, Oct. 2006.

[28] B. Gross, D. Dahl, and L. Nielson, "Physiologic monitoring alarm load on medical/surgical floors of a community hospital,” Biomed Instrum Techno, Suppl, pp. 29-36, Spring 2011.

[29] D.A. Whalen, P.M. Covelle, J.C. Piepenbrink, K. L. Villanova, C. L. Cuneo, and E. H. Awtry, "Novel approach to cardiac alarm managment on telemtry units,” J Cardiovasc Nurs, vol. 29, pp. E13-22, Sep 2014.

[30] American Association of Critical Care Nurses, “AACN practice alert: alarm management,” 2013. [Online]. Available: http://www.aacn.org/wd/practice/docs/practicealerts/alarm-managementpractice-alert.pdf. [Accessed: Oct 3, 2016].

[31] G. Spratt, "Three steps to reduce alarm fatigue and improve patient safety,” AARC Times, vol. 40, pp. 13-16, 2012.

[32] J. R. Blakeman, K. Sarsfield, and K. J. Booker, "Nurses' practices and lead selection in monitoring for myocardial iscehmia: an evidence-based quality improvement project," Dimens Crit Care Nurs, vol 34, pp. 18995, Jul 2015.

[33] The Hospital and Healthsystem Association of Pennsylvania, "Patientand family-centered care: a key elementy in improving quality, safety, perception of care and care outcomes," The Hospital \& Healthsystem Association of Pennsylvania, pp. 1-125, 2013. [Online]. Available: https://www.haponline.org/Portals/0/docs/Initiatives/Improving_Patient Experience/Patient_Family_Centered_Care/HAP_Patient_and_Family_ Centered_Care_Guidebook_July2013.pdf?ver=2013-12-01-183259-640. [Accessed April 6, 2017].

[34] S. Feder, and M. Funk, “Over-monitoring and alarm fatigue: for whom do the bells toll?” Heart Lung, vol. 42, pp. 395-6. Nov. 2013.

[35] E. M. Benjamin, R. A. Klugman, R. Luckmann, D. G. Fairchild, and S. A. Abookire, "Impact of cardiac telemetry monitoring on patient safety and cost,” Am J Manag Care, vol. 19, pp. e225-e232, Nov. 2013.

[36] M.A. Halm, "Hourly rounds: what does the evidence indicate,” Am J Crit Care, vol. 18, pp. 581-84, Nov. 2009.

[37] Healthcare Technology Foundation, “About the HTF,” 2016. [Online]. Available: http://www.thehtf.org/documents/HTF_TrifoldRev2.pdf. [Accessed: Sep. 3, 2017].

[38] ECRI Institute, "Health devices: top 10 health technolgoy hazards for 2012,” Health Devices, vol. 41, pp. 1-17, Nov 2012. 